\title{
ON FUNCTIONAL INEQUALITIES CONNECTED WITH INTERTWINING MAPPINGS
}

\author{
Eun Hwi Lee ANd ICK-Soon Chang
}

\begin{abstract}
For a mapping satisfying the inequality

$$
\|\lambda f(x)+2 \lambda f(y)+2 f(\lambda z)\| \leq\left\|2 f\left(\lambda\left(\frac{x}{2}+y+z\right)\right)\right\|+\phi(x, y, z),
$$
\end{abstract}

we will study the stability problem of this mapping.

\section{Introduction}

In 1940, Ulam [19] proposed the following question concerning the stability of group homomorphisms :

Let $G_{1}$ be a group and let $G_{2}$ be a metric group with the metric d. Given $\epsilon>0$, does there exist a $\delta>0$ such that if a function $h: G_{1} \rightarrow G_{2}$ satisfies the inequality $d(h(x y), h(x) h(y))<\delta$ for all $x, y \in G_{1}$, then there exists a homomorphism $H: G_{1} \rightarrow G_{2}$ with $d(h(x), H(x))<\epsilon$ for all $x \in G_{1}$ ?

In the following year 1941, Hyers [7] answered the problem of Ulam under the assumption that the groups are Banach spaces. A generalized version of the theorem of Hyers for approximately additive mappings was given by Aoki [1] and for the theorem of Hyers for approximately linear mappings it was presented by Rassias [16]. Since then, the stability problems of various functional equations have been extensively investigated by a number of mathematicians (for instances, $[5,6,8,9,10,11,14,15,17,18])$. Moreover, the problems concerned with the generalizations and the applications of the stability to a number of functional equations and mappings have been developed as well.

Received January 22, 2009. Accepted June 4, 2009.

2000 Mathematics Subject Classification: 39B52, 39B72.

Key words and phrases: Stability; Additive mapping; Jordan-von Neumann type functional equation; Intertwining mapping. 
By regarding a large influence of Ulam, Hyers and Rassias on the investigation of stability problems of functional equations the stability phenomenon that was introduced and proved by Rassias [16] in the year 1978 is called the generalized Hyers-Ulam stability or the Hyers-UlamRassias stability.

Let $A$ be a Banach algebra and let $X$ be a weak Banach $A$-bimodule. By a weak Banach $A$-module we mean a Banach space $X$ which is an $A$-bimodule such that for each $a \in A$, the mappings $z \mapsto a z$ and $z \mapsto z a$ defined on $X$ are continuous. A linear mapping $L: A \rightarrow X$ is called intertwining if for each $a \in A$, the mappings $x \mapsto L(a x)-a L(x)$ and $x \mapsto L(x a)-L(x) a$ from $A$ into $X$ are continuous. These mappings were first introduced by Bade and Curtis [2] and were generalized by Laursen [13]. Evidently, every $A$-bimodule homomorphism of $A$ into $X$ and any derivation of $A$ into $X$ are intertwining (cf. $[3,4]$ ).

In this paper, we will take account of the stability problem of a mapping satisfying the inequality

$$
\|\lambda f(x)+2 \lambda f(y)+2 f(\lambda z)\| \leq\left\|2 f\left(\lambda\left(\frac{x}{2}+y+z\right)\right)\right\|+\phi(x, y, z) .
$$

This functional inequality is based on the inequality introduced in [18].

\section{Stability on a Banach algebra}

In this section, let $A$ be a Banach algebra and $X$ be a weak Banach $A$-module, respectively. We also denote by $\mathbb{T}:=\{z \in \mathbb{C}:|z|=1\}$. Let $\phi: A^{3} \rightarrow[0, \infty)$ be a function such that

$$
\sum_{j=0}^{\infty} \frac{1}{2^{j}} \phi\left(-2^{j+1}(x+y), 2^{j} x, 2^{j} y\right)<K
$$

for all $x, y \in A$ and some finite constant $K$.

Theorem 2.1. Let a mapping $f: A \rightarrow X$ satisfy the inequality

$$
\|\lambda f(x)+2 \lambda f(y)+2 f(\lambda z)\| \leq\left\|2 f\left(\lambda\left(\frac{x}{2}+y+z\right)\right)\right\|+\phi(x, y, z)
$$

for all $x, y, z \in A$ and all $\lambda \in \mathbb{T}$. Suppose that for each $a \in A$, mappings

$$
f_{a}(x)=f(a x)-a f(x),{ }_{a} f(x)=f(a x)-f(x) a
$$

are continuous. Then there exists exactly one intertwining mapping $L: A \rightarrow X$ such that

$$
\|L(x)-f(x)\| \leq \rho_{1}(x),
$$


where

$$
\begin{aligned}
\rho_{1}(x)= & \sum_{j=0}^{\infty} \frac{1}{2^{j+1}}\left[\phi\left(-2^{j+1} x, 2^{j} x, 0\right)+\frac{1}{2} \phi\left(0,2^{j+1} x,-2^{j+1} x\right)\right. \\
& \left.+\frac{11}{6} \phi(0,0,0)\right] .
\end{aligned}
$$

Proof. Fix $a \in A$. We demonstrate from (2.1) that

$$
\begin{aligned}
& \left\|\lambda f_{a}(x)+2 \lambda f_{a}(y)+2 f_{a}(\lambda z)\right\| \leq\left\|2 f\left(\lambda a\left(\frac{x}{2}+y+z\right)\right)\right\| \\
& \quad+\|a\|\left\|2 f\left(\lambda\left(\frac{x}{2}+y+z\right)\right)\right\|+\phi(a x, a y, a z)+\|a\| \phi(x, y, z) .
\end{aligned}
$$

Set $\lambda=1$ in (2.4). Then we get

$$
\begin{aligned}
& \left\|f_{a}(x)+2 f_{a}(y)+2 f_{a}(z)\right\| \leq\left\|2 f\left(a\left(\frac{x}{2}+y+z\right)\right)\right\| \\
& \quad+\|a\|\left\|2 f\left(\frac{x}{2}+y+z\right)\right\|+\phi(a x, a y, a z)+\|a\| \phi(x, y, z) .
\end{aligned}
$$

Putting $\lambda=1$ and $x=y=z=0$ in (2.1) gives the inequality

$$
\|f(0)\| \leq \frac{1}{3} \phi(0,0,0) .
$$

By letting $x=y=z=0$ in (2.5), then we arrive at

$$
\left\|f_{a}(0)\right\| \leq\left(\frac{1}{3}+\frac{1}{3}\|a\|\right) \phi(0,0,0) .
$$

And by letting $x=-2 x, y=x$ and $z=0$ in (2.5), we lead to

$$
\begin{aligned}
& \left\|f_{a}(-2 x)+2 f_{a}(x)\right\| \leq \phi(-2 a x, a x, 0)+\|a\| \phi(-2 x, x, 0) \\
& \quad+\left(\frac{4}{3}+\frac{4}{3}\|a\|\right) \phi(0,0,0) .
\end{aligned}
$$

This implies that

$$
\begin{aligned}
& \left\|\frac{1}{2} f_{a}(-2 x)+f_{a}(x)\right\| \leq \frac{1}{2} \phi(-2 a x, a x, 0)+\frac{1}{2}\|a\| \phi(-2 x, x, 0) \\
& \quad+\left(\frac{2}{3}+\frac{2}{3}\|a\|\right) \phi(0,0,0) .
\end{aligned}
$$

Also, by taking $x=0, y=x$ and $z=-x$ in (2.5), we obtain

$$
\begin{aligned}
& \left\|f_{a}(x)+f_{a}(-x)\right\| \leq \frac{1}{2} \phi(0, a x,-a x)+\frac{1}{2}\|a\| \phi(0, x,-x) \\
& +\left(\frac{1}{2}+\frac{1}{2}\|a\|\right) \phi(0,0,0) .
\end{aligned}
$$


Combine (2.6) and (2.7) to find that

$$
\begin{aligned}
& \left\|\frac{f_{a}\left(2^{l} x\right)}{2^{l}}-\frac{f_{a}\left(2^{m} x\right)}{2^{m}}\right\| \leq \sum_{j=l}^{m-1}\left\|\frac{f_{a}\left(2^{j} x\right)}{2^{j}}-\frac{f_{a}\left(2^{j+1} x\right)}{2^{j+1}}\right\| \\
& \leq \sum_{j=l}^{m-1}\left[\left\|\frac{f_{a}\left(2^{j} x\right)}{2^{j}}+\frac{f_{a}\left(-2^{j+1} x\right)}{2^{j+1}}\right\|+\left\|\frac{f_{a}\left(2^{j+1} x\right)}{2^{j+1}}+\frac{f_{a}\left(-2^{j+1} x\right)}{2^{j+1}}\right\|\right] \\
& \leq \sum_{j=l}^{m-1} \frac{1}{2^{j+1}}\left[\phi\left(-2^{j+1} a x, 2^{j} a x, 0\right)+\frac{1}{2} \phi\left(0,2^{j+1} a x,-2^{j+1} a x\right)\right. \\
& \quad+\|a\| \phi\left(-2^{j+1} x, 2^{j} x, 0\right)+\frac{1}{2}\|a\| \phi\left(0,2^{j+1} x,-2^{j+1} x\right) \\
& \left.\quad+\left(\frac{11}{6}+\frac{11}{6}\|a\|\right) \phi(0,0,0)\right]
\end{aligned}
$$

for all nonnegative integers $m$ and $l$ with $m>l$ and all $x \in A$. This means that the sequence $\left\{f_{a}\left(2^{n} x\right) / 2^{n}\right\}$ is a Cauchy sequence. Since $X$ is complete, the sequence $\left\{f_{a}\left(2^{n} x\right) / 2^{n}\right\}$ converges. Thus we may define the mapping $L_{a}: A \rightarrow X$ by $L_{a}(x):=\lim _{n \rightarrow \infty} f_{a}\left(2^{n} x\right) / 2^{n}$ for all $x \in A$. In particular, by setting $l=0$ and passing the limit as $m \rightarrow \infty$, we have

$$
\left\|f_{a}(x)-L_{a}(x)\right\| \leq \sigma_{1}(x),
$$

where

$$
\begin{aligned}
\sigma_{1}(x)= & \sum_{j=0}^{\infty} \frac{1}{2^{j+1}}\left[\phi\left(-2^{j+1} a x, 2^{j} a x, 0\right)+\frac{1}{2} \phi\left(0,2^{j+1} a x,-2^{j+1} a x\right)\right. \\
& +\|a\| \phi\left(-2^{j+1} x, 2^{j} x, 0\right)+\frac{1}{2}\|a\| \phi\left(0,2^{j+1} x,-2^{j+1} x\right) \\
& \left.+\left(\frac{11}{6}+\frac{11}{6}\|a\|\right) \phi(0,0,0)\right] .
\end{aligned}
$$

First of all, we note due to (2.7) that

$$
\begin{aligned}
& \left\|L_{a}(x)+L_{a}(-x)\right\|=\lim _{n \rightarrow \infty} \frac{1}{2^{n}}\left\|f_{a}\left(2^{n} x\right)+f_{a}\left(-2^{n} x\right)\right\| \\
& \leq \lim _{n \rightarrow \infty}\left[\frac{1}{2^{n+1}} \phi\left(0,2^{n} a x,-2^{n} a x\right)+\frac{1}{2^{n+1}}\|a\| \phi\left(0,2^{n} x,-2^{n} x\right)\right. \\
& \left.\quad+\frac{1}{2^{n}}\left(\frac{1}{2}+\frac{1}{2}\|a\|\right) \phi(0,0,0)\right]=0,
\end{aligned}
$$

from the assumption of $\phi$. So we have $L_{a}(-x)=-L_{a}(x)$. 
Now, we claim that the mapping $L_{a}: A \rightarrow X$ is an additive mapping: Use (2.5), (2.6) and (2.7) to obtain that

$$
\begin{aligned}
&\left\|L_{a}(x)+L_{a}(y)-L_{a}(x+y)\right\| \\
&=\lim _{n \rightarrow \infty} \frac{1}{2^{n}}\left\|f_{a}\left(2^{n} x\right)+f_{a}\left(2^{n} y\right)+f_{a}\left(2^{n}(-x-y)\right)\right\| \\
& \leq \lim _{n \rightarrow \infty}\left\{\frac{1}{2^{n}}\left\|\frac{1}{2} f_{a}\left(2^{n+1}(-x-y)\right)+f_{a}\left(2^{n} x\right)+f_{a}\left(2^{n} y\right)\right\|\right. \\
&+\frac{1}{2^{n}}\left\|f_{a}\left(2^{n}(-x-y)\right)+\frac{1}{2} f_{a}\left(2^{n+1}(x+y)\right)\right\| \\
&\left.+\frac{1}{2^{n+1}}\left\|f_{a}\left(2^{n+1}(-x-y)\right)+f_{a}\left(2^{n+1}(x+y)\right)\right\|\right\} \\
& \leq \lim _{n \rightarrow \infty}\left\{\frac { 1 } { 2 ^ { n + 1 } } \left[\phi\left(2^{n+1} a(-x-y), 2^{n} a x, 2^{n} a y\right)\right.\right. \\
&\left.+\|a\| \phi\left(2^{n+1}(-x-y), 2^{n} x, 2^{n} y\right)\right] \\
&+\frac{1}{2^{n+1}}\left[\phi\left(2^{n+1} a(x+y), 2^{n} a(-x-y), 0\right)\right. \\
&\left.+\frac{1}{2} \phi\left(0,2^{n+1} a(-x-y), 2^{n+1} a(x+y)\right)\right] \\
&+\frac{1}{2^{n+1}}\|a\|\left[\phi\left(2^{n+1}(x+y), 2^{n}(-x-y), 0\right)\right. \\
&\left.+\frac{1}{2} \phi\left(0,2^{n+1}(-x-y), 2^{n+1}(x+y)\right)\right] \\
&\left.+\frac{1}{2^{n}}\left(\frac{5}{4}+\frac{5}{4}\|a\|\right) \phi(0,0,0)\right\}=0,
\end{aligned}
$$

from the assumption of $\phi$.

In order to prove that the mapping $L_{a}(x)$ is unique, let us assume that $S_{a}: A \rightarrow X$ be another additive mapping satisfying (2.8). Then it follows that

$$
\begin{aligned}
\left\|L_{a}(x)-S_{a}(x)\right\| & =\frac{1}{2^{n}}\left\|L_{a}\left(2^{n} x\right)-S_{a}\left(2^{n} x\right)\right\| \\
& \leq \frac{1}{2^{n}}\left[\left\|L_{a}\left(2^{n} x\right)-f_{a}\left(2^{n} x\right)\right\|+\left\|S_{a}\left(2^{n} x\right)-f_{a}\left(2^{n} x\right)\right\|\right] \\
& \leq \frac{1}{2^{n-1}} \sigma_{1}\left(2^{n} x\right) \rightarrow 0
\end{aligned}
$$


as $n \rightarrow \infty$, for all $x \in A$, because

$$
\begin{aligned}
\lim _{n \rightarrow \infty} & \frac{1}{2^{n-1}} \sigma_{1}\left(2^{n} x\right) \\
= & \lim _{n \rightarrow \infty} \sum_{j=n}^{\infty} \frac{1}{2^{j+1}}\left[\phi\left(-2^{j+1} a x, 2^{j} a x, 0\right)+\frac{1}{2} \phi\left(0,2^{j+1} a x,-2^{j+1} a x\right)\right. \\
& +\|a\| \phi\left(-2^{j+1} x, 2^{j} x, 0\right)+\frac{1}{2}\|a\| \phi\left(0,2^{j+1} x,-2^{j+1} x\right) \\
& \left.+\left(\frac{11}{6}+\frac{11}{6}\|a\|\right) \phi(0,0,0)\right]=0 .
\end{aligned}
$$

Consequently, we conclude that there exists exactly one additive mapping $L_{a}: A \rightarrow X$ satisfying (2.8).

On the other hand, we have by letting $x=0, y=x$ and $z=-x$ in (2.5) that

$$
\begin{aligned}
& \left\|\lambda f_{a}(x)+f_{a}(-\lambda x)\right\| \leq \frac{1}{2} \phi(0, a x,-a x)+\frac{1}{2}\|a\| \phi(0, x,-x) \\
& \quad+\left(\frac{1}{2}+\frac{1}{2}\|a\|\right) \phi(0,0,0) .
\end{aligned}
$$

Replace $x$ by $2^{n} x$ in (2.9) and divide by $2^{n}$ to get

$$
\begin{aligned}
& \left\|\lambda \frac{f_{a}\left(2^{n} x\right)}{2^{n}}+\frac{f_{a}\left(-2^{n} \lambda x\right)}{2^{n}}\right\| \leq \frac{1}{2^{n+1}} \phi\left(0,2^{n} a x,-2^{n} a x\right) \\
& \quad+\frac{1}{2^{n+1}}\|a\| \phi\left(0,2^{n} x,-2^{n} x\right)+\frac{1}{2^{n}}\left(\frac{1}{2}+\frac{1}{2}\|a\|\right) \phi(0,0,0) .
\end{aligned}
$$

By taking $n \rightarrow \infty$, we find that $L_{a}(\lambda x)=\lambda L_{a}(x)$ for all $x \in A$ and all $\lambda \in \mathbb{T}$, by assumption of $\phi$. Here and now, let $\mu \in \mathbb{C}$ be a nonzero and $M$ an integer greater than $4|\mu|$. Then $\left|\frac{\mu}{M}\right|<\frac{1}{4}<1-\frac{2}{3}$. According to the theorem in [12], we see that there exist elements $\lambda_{1}, \lambda_{2}, \lambda_{3} \in \mathbb{T}$ such that $3 \frac{\mu}{M}=\lambda_{1}+\lambda_{2}+\lambda_{3}$. Observe that $L_{a}\left(\frac{1}{3} x\right)=\frac{1}{3} L_{a}(x)$ for all $x \in A$. Thus it follows that

$$
\begin{aligned}
& L_{a}(\mu x)=L_{a}\left(\frac{M}{3} \cdot 3 \frac{\mu}{M} x\right)=M L_{a}\left(\frac{1}{3} \cdot 3 \frac{\mu}{M} x\right)=\frac{M}{3} L_{a}\left(3 \frac{\mu}{M} x\right) \\
& \quad=\frac{M}{3} L_{a}\left(\lambda_{1} x+\lambda_{2} x+\lambda_{3} x\right)=\frac{M}{3}\left(L_{a}\left(\lambda_{1} x\right)+L_{a}\left(\lambda_{2} x\right)+L_{a}\left(\lambda_{3} x\right)\right) \\
& \quad=\frac{M}{3}\left(\lambda_{1}+\lambda_{2}+\lambda_{3}\right) L_{a}(x)=\frac{M}{3} \cdot 3 \frac{\mu}{M} \cdot L_{a}(x)=\mu L_{a}(x)
\end{aligned}
$$

for all $x \in A$. Hence, by the additivity of $L_{a}$, we obtain that

$$
L_{a}(\zeta x+\eta y)=\zeta L_{a}(x)+\eta L_{a}(y)
$$


for all $x \in A$ and for all $\zeta, \eta \in \mathbb{C}$ with $\zeta \neq 0, \eta \neq 0$. In particular, $L_{a}(0 x)=0=0 L_{a}(x)$. Therefore, $L_{a}$ is a linear mapping.

Now, we need to show that the mapping $L_{a}$ is continuous: Suppose that $f_{a}$ is continuous at a point $x_{0} \in A$ and there exists a point such that $L_{a}$ is not continuous at this point. Then there exist a positive number $\eta$ and a sequence $\left\{x_{n}\right\}$ in $A$ such that $\lim _{n \rightarrow \infty} x_{n}=0$ and $\left\|L_{a}\left(x_{n}\right)\right\|>\eta$. Set $\epsilon>\frac{3 \alpha}{\eta}$, where $\sigma_{1}(x)<\alpha$ for all $x \in A$. Note that

$$
\left\|L_{a}\left(\varepsilon x_{n}+x_{0}\right)-L_{a}\left(x_{0}\right)\right\|=\left\|L_{a}\left(\varepsilon x_{n}\right)\right\|>3 \alpha
$$

for all $n$. Since $\lim _{n \rightarrow \infty} f_{a}\left(\varepsilon x_{n}+x_{0}\right)=f_{a}\left(x_{0}\right)$, there is an integer $N$ such that for all $n>N$

$$
\begin{gathered}
\left\|L_{a}\left(\varepsilon x_{n}+x_{0}\right)-L_{a}\left(x_{0}\right)\right\| \leq\left\|L_{a}\left(\varepsilon x_{n}+x_{0}\right)-f_{a}\left(\varepsilon x_{n}+x_{0}\right)\right\| \\
\quad+\left\|f_{a}\left(\varepsilon x_{n}+x_{0}\right)-f_{a}\left(x_{0}\right)\right\|+\left\|f_{a}\left(x_{0}\right)-L_{a}\left(x_{0}\right)\right\|<3 \alpha,
\end{gathered}
$$

which is a contradiction.

Let $\lambda=1$ in (2.1). Following the similar method as the previous proof, there is exactly one additive mapping $L: A \rightarrow X$ satisfying the inequality (2.3), where $L(x):=\lim _{n \rightarrow \infty} f\left(2^{n} x\right) / 2^{n}$ for all $x \in A$.

We also imply by putting $x=0, y=x$ and $z=-x$ in (2.1) that

$$
\|\lambda f(x)+f(-\lambda x)\| \leq \frac{1}{2} \phi(0, x,-x)+\frac{1}{2} \phi(0,0,0) .
$$

Replace $x$ by $2^{n} x$ in (2.10) and divide by $2^{n}$ to get

$$
\left\|\lambda \frac{f\left(2^{n} x\right)}{2^{n}}+\frac{f\left(-2^{n} \lambda x\right)}{2^{n}}\right\| \leq \frac{1}{2^{n+1}} \phi\left(0,2^{n} x,-2^{n} x\right)+\frac{1}{2^{n+1}} \phi(0,0,0) .
$$

Taking the limit as $n \rightarrow \infty$, we have $L(\lambda x)=\lambda L(x)$ for all $x \in A$ and all $\lambda \in \mathbb{T}$, from assumption of $\phi$. By the same reasoning as the above proof, $L$ is a linear mapping.

Let us replace $x$ by $2^{n} x$ in (2.2) and then divide by $2^{n}$. Then, by utilizing the definition of $L_{a}$ and $L$, we yield that

$$
L_{a}(x)=\lim _{n \rightarrow \infty} \frac{f_{a}\left(2^{n} x\right)}{2^{n}}=\lim _{n \rightarrow \infty}\left[\frac{f\left(2^{n} a x\right)}{2^{n}}-a \frac{f\left(2^{n} x\right)}{2^{n}}\right]=L(a x)-a L(x) .
$$

In similar manner, one can show that for each $a \in A$, a linear mapping ${ }_{a} L: A \rightarrow X$ defined by ${ }_{a} L(x):=\lim _{n \rightarrow \infty} f\left(2^{n} x\right) / 2^{n}$ is continuous on $A$ and ${ }_{a} L(x)=L(x a)-L(x) a$.

Thus $L$ is an intertwining mapping. The proof of Theorem is complete. 
Corollary 2.2. Let $p, q<1$ and $r<1$ be real number and let a mapping $f: A \rightarrow X$ satisfy the inequality

$$
\begin{aligned}
& \|\lambda f(x)+2 \lambda f(y)+2 f(\lambda z)\| \leq\left\|2 f\left(\lambda\left(\frac{x}{2}+y+z\right)\right)\right\| \\
& \quad+\theta\left(\|x\|^{p}+\|y\|^{q}+\|z\|^{r}\right)
\end{aligned}
$$

for all $x, y, z \in A$ and all $\lambda \in \mathbb{T}$. Suppose that for each $a \in A$, the mappings (2.2) are continuous. Then there exists exactly one intertwining mapping $L: A \rightarrow X$ such that

$$
\|L(x)-f(x)\| \leq \theta\left[\frac{2^{p}}{2-2^{p}}\|x\|^{p}+\frac{1+2^{q-1}}{2-2^{q}}\|x\|^{q}+\frac{2^{r}}{2-2^{r}}\|x\|^{r}\right] .
$$

Corollary 2.3. Let a mapping $f: A \rightarrow X$ satisfy the inequality

$$
\|\lambda f(x)+2 \lambda f(y)+2 f(\lambda z)\| \leq\left\|2 f\left(\lambda\left(\frac{x}{2}+y+z\right)\right)\right\|+\theta
$$

for all $x, y, z \in A$ and all $\lambda \in \mathbb{T}$. Suppose that for each $a \in A$, the mappings (2.2) are continuous. Then there exists exactly one intertwining mapping $L: A \rightarrow X$ such that

$$
\|L(x)-f(x)\| \leq \frac{10}{3} \theta .
$$

Theorem 2.4. Let a mapping $f: A \rightarrow X$ satisfy the inequality (2.1). Suppose that for each $a \in A$, the mappings (2.2) are continuous. Then there exists exactly one intertwining mapping $L: A \rightarrow X$ such that

$$
\left\|L(x)-\frac{f(x)-f(-x)}{2}\right\| \leq \rho_{2}(x),
$$

where

$\rho_{2}(x)=\sum_{j=0}^{\infty} \frac{1}{2^{j+2}}\left[\phi\left(-2^{j+1} x, 2^{j} x, 0\right)+\phi\left(2^{j+1} x,-2^{j} x, 0\right)+\frac{8}{3} \phi(0,0,0)\right]$.

Proof. For each $a \in A$, we define a mapping $g_{a}: A \rightarrow X$ by

$$
g_{a}(x)=\frac{f_{a}(x)-f_{a}(-x)}{2}
$$

for all $x \in A$. Due to (2.6), we obtain

$$
\begin{aligned}
& \left\|\frac{1}{2} f_{a}(x)+f_{a}\left(-\frac{x}{2}\right)\right\| \leq \frac{1}{2} \phi\left(a x,-\frac{a x}{2}, 0\right)+\frac{1}{2}\|a\| \phi\left(x,-\frac{x}{2}, 0\right) \\
& \quad+\left(\frac{2}{3}+\frac{2}{3}\|a\|\right) \phi(0,0,0) .
\end{aligned}
$$


So we have by (2.12)

$$
\begin{aligned}
& \left\|\frac{g_{a}\left(2^{l} x\right)}{2^{l}}-\frac{g_{a}\left(2^{m} x\right)}{2^{m}}\right\| \leq \sum_{j=l}^{m-1}\left\|\frac{g_{a}\left(2^{j} x\right)}{2^{j}}-\frac{g_{a}\left(2^{j+1} x\right)}{2^{j+1}}\right\| \\
& \leq \sum_{j=l}^{m-1} \frac{1}{2^{j+2}}\left[\phi\left(-2^{j+1} a x, 2^{j} a x, 0\right)+\phi\left(2^{j+1} a x,-2^{j} a x, 0\right)\right. \\
& \quad+\|a\| \phi\left(-2^{j+1} x, 2^{j} x, 0\right)+\|a\| \phi\left(2^{j+1} x,-2^{j} x, 0\right) \\
& \left.\quad+\left(\frac{8}{3}+\frac{8}{3}\|a\|\right) \phi(0,0,0)\right]
\end{aligned}
$$

for all nonnegative integers $m$ and $l$ with $m>l$ and all $x \in A$. It means that the sequence $\left\{g_{a}\left(2^{n} x\right) / 2^{n}\right\}$ is a Cauchy sequence. Since $X$ is complete, the sequence $\left\{g_{a}\left(2^{n} x\right) / 2^{n}\right\}$ converges. So we can define the mapping $L_{a}: A \rightarrow X$ by $L_{a}(x):=\lim _{n \rightarrow \infty} g_{a}\left(2^{n} x\right) / 2^{n}$ for all $x \in A$. Furthermore, by letting $l=0$ and passing the limit as $m \rightarrow \infty$, we get

$$
\left\|g_{a}(x)-L_{a}(x)\right\| \leq \sigma_{2}(x),
$$

where

$$
\begin{aligned}
& \sigma_{2}(x)=\sum_{j=0}^{\infty} \frac{1}{2^{j+2}}\left[\phi\left(-2^{j+1} a x, 2^{j} a x, 0\right)+\phi\left(2^{j+1} a x,-2^{j} a x, 0\right)\right. \\
& \left.+\|a\| \phi\left(-2^{j+1} x, 2^{j} x, 0\right)+\|a\| \phi\left(2^{j+1} x,-2^{j} x, 0\right)+\left(\frac{8}{3}+\frac{8}{3}\|a\|\right) \phi(0,0,0)\right] .
\end{aligned}
$$

Next, we claim that the mapping $L_{a}: A \rightarrow X$ is an additive mapping: We first note that $L_{a}(-x)=-L_{a}(x)$, because $g_{a}$ is an odd function. If use the same argument as in the proof of Theorem 2.1, then we deduce

$$
\lim _{n \rightarrow \infty} \frac{1}{2^{n}}\left\|f_{a}\left(2^{n} x\right)+f_{a}\left(2^{n} y\right)+f_{a}\left(2^{n}(-x-y)\right)\right\|=0 .
$$

From this, we can show that

$$
\begin{aligned}
& \left\|L_{a}(x)+L_{a}(y)-L_{a}(x+y)\right\| \\
& \quad=\lim _{n \rightarrow \infty} \frac{1}{2^{n}}\left\|g_{a}\left(2^{n} x\right)+g_{a}\left(2^{n} y\right)+g_{a}\left(2^{n}(-x-y)\right)\right\| \\
& \leq \lim _{n \rightarrow \infty}\left[\frac{1}{2^{n+1}}\left\|f_{a}\left(2^{n} x\right)+f_{a}\left(2^{n} y\right)+f_{a}\left(2^{n}(-x-y)\right)\right\|\right. \\
& \left.\quad+\frac{1}{2^{n+1}}\left\|f_{a}\left(-2^{n} x\right)+f_{a}\left(-2^{n} y\right)+f_{a}\left(2^{n}(x+y)\right)\right\|\right]=0 .
\end{aligned}
$$

Now, to prove the uniqueness of the mapping $L_{a}(x)$, let us assume that $T_{a}: A \rightarrow X$ be another additive mapping satisfying (2.13). Then 
we find that

$$
\begin{aligned}
\lim _{n \rightarrow \infty} & \frac{1}{2^{n-1}} \sigma_{2}\left(2^{n} x\right) \\
= & \lim _{n \rightarrow \infty} 2 \sum_{j=n}^{\infty} \frac{1}{2^{j+2}}\left[\phi\left(-2^{j+1} a x, 2^{j} a x, 0\right)+\phi\left(2^{j+1} a x,-2^{j} a x, 0\right)\right. \\
& +\|a\| \phi\left(-2^{j+1} x, 2^{j} x, 0\right)+\|a\| \phi\left(2^{j+1} x,-2^{j} x, 0\right) \\
& \left.+\left(\frac{8}{3}+\frac{8}{3}\|a\|\right) \phi(0,0,0)\right]=0,
\end{aligned}
$$

which implies that

$$
\begin{aligned}
\left\|L_{a}(x)-T_{a}(x)\right\| & =\frac{1}{2^{n}}\left\|L_{a}\left(2^{n} x\right)-T_{a}\left(2^{n} x\right)\right\| \\
& \leq \frac{1}{2^{n}}\left[\left\|L_{a}\left(2^{n} x\right)-f_{a}\left(2^{n} x\right)\right\|+\left\|T_{a}\left(2^{n} x\right)-f_{a}\left(2^{n} x\right)\right\|\right] \\
& \leq \frac{1}{2^{n-1}} \sigma_{2}\left(2^{n} x\right) \rightarrow 0
\end{aligned}
$$

as $n \rightarrow \infty$, for all $x \in A$.

So that there exists exactly one additive mapping $L_{a}: A \rightarrow X$ satisfying (2.13).

By (2.9), we have

$$
\begin{aligned}
&\left\|\lambda g_{a}(x)+g_{a}(-\lambda x)\right\| \\
& \leq \frac{1}{2}\left\|\lambda f_{a}(x)+f_{a}(-\lambda x)\right\|+\frac{1}{2}\left\|\lambda f_{a}(-x)+f_{a}(\lambda x)\right\| \\
& \leq \frac{1}{4} \phi(0, a x,-a x)+\frac{1}{4}\|a\| \phi(0, x,-x)+\frac{1}{4} \phi(0,-a x, a x) \\
&+\frac{1}{4}\|a\| \phi(0,-x, x)+\left(\frac{1}{2}+\frac{1}{2}\|a\|\right) \phi(0,0,0) .
\end{aligned}
$$

Substitute $x:=2^{n} x$ in the above inequality and multiply $\frac{1}{2^{n}}$ to obtain

$$
\begin{aligned}
\| \lambda & \frac{g_{a}\left(2^{n} x\right)}{2^{n}}+\frac{g_{a}\left(-2^{n} \lambda x\right)}{2^{n}} \| \\
\leq & \frac{1}{2^{n+2}} \phi\left(0,2^{n} a x,-2^{n} a x\right)+\frac{1}{2^{n+2}}\|a\| \phi\left(0,2^{n} x,-2^{n} x\right) \\
& +\frac{1}{2^{n+2}} \phi\left(0,-2^{n} a x, 2^{n} a x\right)+\frac{1}{2^{n+2}}\|a\| \phi\left(0,-2^{n} x, 2^{n} x\right) \\
& +\frac{1}{2^{n}}\left(\frac{1}{2}+\frac{1}{2}\|a\|\right) \phi(0,0,0) .
\end{aligned}
$$


In this inequality, let $n \rightarrow \infty$. Then $L_{a}(\lambda x)=\lambda L_{a}(x)$, where $x \in A$ and $\lambda \in \mathbb{T}$. As the proof of Theorem 2.1, we can show that $L_{a}$ is a linear mapping.

On the other hand, continuity of $f_{a}$ at $x_{0} \in A$ guarantees continuity of $f_{a}$ at $-x_{0} \in A$, since $f_{a}$ is continuous on $A$. Thus, by the definition of $g_{a}$, we see that $g_{a}$ is continuous at $x_{0} \in A$.

It remains to show that $L_{a}$ is continuous at $x_{0} \in A$. If we use the contrapositive, then, as in the proof of Theorem 2.1, we can prove that $L_{a}$ is continuous at $x_{0} \in A$.

Put $\lambda=1$ in (2.1). Similarly, as we did in the precedent proof, there exists exactly one additive mapping $L: A \rightarrow X$ satisfying the inequality (2.11), where

$$
L(x)=\lim _{n \rightarrow \infty} \frac{g\left(2^{n} x\right)}{2^{n}}, g(x)=\frac{f(x)-f(-x)}{2}
$$

for all $x \in A$.

Now it follows from (2.10) that

$$
\begin{gathered}
\|\lambda g(x)+g(-\lambda x)\| \leq \frac{1}{2}\|\lambda f(x)+f(-\lambda x)\|+\frac{1}{2}\|\lambda f(-x)+f(\lambda x)\| \\
\leq \leq \frac{1}{4} \phi(0, x,-x)+\frac{1}{4} \phi(0,-x, x)+\frac{1}{2} \phi(0,0,0) .
\end{gathered}
$$

Replacing $x$ by $2^{n} x$ in (2.14) and dividing by $2^{n}$, we yield that

$$
\begin{aligned}
& \left\|\lambda \frac{g\left(2^{n} x\right)}{2^{n}}+\frac{g\left(-2^{n} \lambda x\right)}{2^{n}}\right\| \leq \frac{1}{2^{n+2}} \phi\left(0,2^{n} x,-2^{n} x\right)+\frac{1}{2^{n+2}} \phi\left(0,-2^{n} x, 2^{n} x\right) \\
& \quad+\frac{1}{2^{n+1}} \phi(0,0,0) .
\end{aligned}
$$

Passing the limit, we see that $L(\lambda x)=\lambda L(x)$ for all $x \in A$ and all $x \in \mathbb{T}$, by assumption of $\phi$. By the same way as the proof of Theorem 2.1, we have

$$
L(\zeta x+\eta y)=\zeta L(x)+\eta L(y)
$$

for all $x \in A$ and all $\zeta, \eta \in \mathbb{C}$, that is, $L$ is a linear mapping.

Replacing $x$ by $2^{n} x$ in (2.2) and then dividing by $2^{n}$, it follows from the definition of $L_{a}$ and $L$ that

$$
L_{a}(x)=\lim _{n \rightarrow \infty} \frac{g_{a}\left(2^{n} x\right)}{2^{n}}=\lim _{n \rightarrow \infty}\left[\frac{g\left(2^{n} a x\right)}{2^{n}}-a \frac{g\left(2^{n} x\right)}{2^{n}}\right]=L(a x)-a L(x) .
$$

In similar fashion, we can show that for each $a \in A$, the linear mapping ${ }_{a} L: A \rightarrow X$ defined by ${ }_{a} L(x):=\lim _{n \rightarrow \infty} \frac{1}{2^{n}} g_{a}\left(2^{n} x\right)$ is continuous on $A$ and ${ }_{a} L=L(x a)-L(x) a$. 
So $L$ is an intertwining mapping. This completes the proof of the theorem.

Corollary 2.5. Let $p, q<1$ and $r<1$ be real number and let a mapping $f: A \rightarrow X$ satisfy the inequality

$$
\begin{aligned}
& \|\lambda f(x)+2 \lambda f(y)+2 f(\lambda z)\| \leq\left\|2 f\left(\lambda\left(\frac{x}{2}+y+z\right)\right)\right\| \\
& \quad+\theta\left(\|x\|^{p}+\|y\|^{q}+\|z\|^{r}\right)
\end{aligned}
$$

for all $x, y, z \in A$ and all $\lambda \in \mathbb{T}$. Suppose that for each $a \in A$, the mappings (2.2) are continuous. Then there exists exactly one intertwining mapping $L: A \rightarrow X$ such that

$$
\left\|L(x)-\frac{f(x)-f(-x)}{2}\right\| \leq \theta\left[\frac{2^{p}}{2-2^{p}}\|x\|^{p}+\frac{2^{q}}{2-2^{q}}\|x\|^{q}\right] .
$$

Corollary 2.6. Let a mapping $f: A \rightarrow X$ satisfy the inequality

$$
\|\lambda f(x)+2 \lambda f(y)+2 f(\lambda z)\| \leq\left\|2 f\left(\lambda\left(\frac{x}{2}+y+z\right)\right)\right\|+\theta
$$

for all $x, y, z \in A$ and all $\lambda \in \mathbb{T}$. Suppose that for each $a \in A$, the mappings (2.2) are continuous. Then there exists exactly one intertwining mapping $L: A \rightarrow X$ such that

$$
\left\|L(x)-\frac{f(x)-f(-x)}{2}\right\| \leq \frac{7}{3} \theta .
$$

Acknowledgement. The authors would like to thank referees for their valuable comments. The second author dedicates this paper to his late father.

\section{References}

[1] T. Aoki, On the stability of the linear transformation in Banach spaces, J. Math. Soc. Japan 2 (1950), 64-66.

[2] W.G. Bade and P.C. Curtis Jr., Prime ideals and automatic continuity problems in Banach modules over $C^{*}$-algebras, J. Funct Anal. 29 (1978), 88-103.

[3] H.G. Dales, Banach algebras and automatic continuity, London Math. Soc. Monogr(N.S). 24, Clarendon Press, Oxford University Press, Oxford 2000.

[4] H.G. Dales and A.R. Vilena, Continuity of derivations, intertwining maps, and cocycles from Banach algebras, J. London Math. Soc. 63 (2001), 215-225.

[5] W. Fechner, Stability of a functional inequality associated with the Jordan-von Neumann functional equation, Aequationes Math.71 (2006), 149-161.

[6] Z. Gajda, On stability of additive mappings, Internat. J. Math. Math. Sci. 14 (1991), 431-434.

[7] D.H. Hyers, On the stability of the linear functional equation, Proc. Nat. Acad. Sci. U.S.A. 27 (1941), 222-224. 
[8] D.H. Hyers, G. Isac and Th.M. Rassias, Stability of functional equations in several variablers, Birkhäuser, Basel, (1998).

[9] K.W. Jun and H.-M. Kim, Stability problem of Ulam for generalized forms of Cauchy functional equation, J. Math. Anal. Appl. 312 (2005), 535-547.

[10] K.W. Jun and Y.H. Lee, A generalization of the Hyers-Ulam-Rassias stability of the Pexiderized quadratic equations, J. Math. Anal. Appl. 297 (2004), 70-86.

[11] S.-M. Jung, Hyers-Ulam stability of a system of first order linear differential equations with constant coefficients, J. Math. Anal. Appl. 320 (2006), 549-561.

[12] R.V. Kadison and G. Pederson, Means and convex combinations of unitary operators, Math. Scand. 57 (1985), 249-266.

[13] K.B. Laursen, Automatic continuity of generalized intertwining operators, Dissertationes Math. (Rozprawy Mat.) 189 (1981).

[14] C. Park, Y. Cho and M. Han, Functional inequalities associated with JordanVon Neumann type additive functional equations, J. Inequal. Appl., 2007 (2007), Article ID 41820, 13 pages.

[15] J.M. Rassias, On approximation of approximately linear mappings by linear mappings, J. Funct. Anal. 46 (1982), 126-130.

[16] Th.M. Rassias, On the stability of the linear mapping in Banach spaces, Proc. Amer. Math. Soc. 72 (1978), 297-300.

[17] Th.M. Rassias and P. Semrl, On the behavior of mappings which do not satisfy Hyers-Ulam stability, Proc. Amer. Math. Soc. 114 (1992), 989-993.

[18] J. Roh and I.-S. Chang, Functional inequalities associated with additive mappings, Abstr. Appl. Anal. 2008 (2008), Article ID 136592, 1-10.

[19] S.M. Ulam, A collection of the mathematical problems, Interscience Publ. New York, (1960).

Eun Hwi Lee

Department of Mathematics,

Jeonju University,

Jeonju 560-759, Republic of Korea

E-mail: ehl@jj.ac.kr

Ick-Soon Chang

Department of Mathematics,

Mokwon University,

Daejeon 302-729, Republic of Korea

E-mail: ischang@mokwon.ac.kr 